\title{
Papillary Muscle Function Does Not Predict Mitral Regurgitation in Patients with Normal Left Ventricular Systolic Function: A Transesophageal Echocardiographic Study
}

\section{-PM function and Mitral Regurgitation}

\author{
Ernest C. Madu ${ }^{1,2}$, Dainia S. Baugh ${ }^{1,2}$, Edwin Tulloch-Reid ${ }^{1,2}$, Chiranjivi Potu ${ }^{1,2}$ \\ ${ }^{1}$ Department of Medicine, Division of Cardiovascular Medicine, Heart Institute of the Caribbean, Kingston, Jamaica; ${ }^{2} \mathrm{Centre}$ of Ex- \\ cellence for Cardiovascular Diseases and Sports Physiology, University of Technology, Kingston, Jamaica. \\ Email:emadu@caribbeanheart.com
}

Received March $3^{\text {rd }} 2011$; revised March 31 ${ }^{\text {st }} 2011$; accepted April $29^{\text {th }} 2011$.

\begin{abstract}
Objective: To evaluate LV papillary muscles (PM) function using transesophageal echocardiography (TEE), and to determine the relationship between PM function and mitral regurgitation in patients with normal left ventricular systolic function. Design: TEE examinations were prospectively performed. End diastolic and end systolic PM lengths were obtained from the transgastric long axis views and fractional systolic shortening (FS) was calculated. LV ejection fraction was determined using modified Simpson rule and mitral regurgitation was determined using regurgitant jet area by color flow. Setting: Tertiary Center. Patients: 85 consecutive adult patients (51 with mitral regurgitation and 34 without) with normal $L V$ chamber dimensions and LV systolic function, meeting enrollment criteria. Results: The \% FS in patients with mitral regurgitation was $21.7 \% \pm 3.6 \%$ for anterior $P M(A P M)$ and $18.7 \% \pm 4.6 \%$ for posterior $P M$ $(P P M)$. In those without mitral regurgitation, the values were as follows; $22.6 \% \pm 5.4 \%(A P M)$ and $19.5 \% \pm 3.8 \%$ $(P P M)$. In a subgroup of patients with severe mitral regurgitation $(n=23)$, the values for PM FS were $20.3 \pm 6.8(A P M)$ and $18.4 \% \pm 6.9 \%(P P M)$. There was no statistically significant difference in PM fractional shortening between the groups. Anterior papillary muscle length was longer in those patients with mitral regurgitation compared to those without [(End-diastolic length (cm): $3.38 \pm 0.61 v 2.88 \pm 0.47(p: 0.008)$ and end-systolic length of $2.46 \pm 0.51 v 2.17 \pm$ 0.33 ( $p: 0.04)$ ]. These differences are more pronounced in those with severe mitral regurgitation ( $p: 0.002$ and 0.004 for EDL and ESL respectively. Conclusion: In patients with normal LVEF, PM contraction is similar in those with and without MR. In patients with MR however, anterior PM length (ED \& ES) is significantly increased. Our data suggests that in patients with normal LVEF, PM dysfunction appears to play no significant role in the causation of MR. Anterior papillary muscle length however, appears to be a major determinant of mitral regurgitation in such patients.
\end{abstract}

Keywords: Mitral Regurgitation, TEE, Papillary Muscle, LV Function

\section{Introduction}

The role of papillary muscle dysfunction in the causation of mitral regurgitation has been the subject of intense debate and controversy over the years. However no prior systematic study in humans has verified the true relationship between papillary muscle function and mitral regurgitation in patients with normal $\mathrm{LV}$ function.

Many anatomic and experimental studies of papillary muscles have been reported [1-4] including a few clinical investigations of human papillary muscles using two- dimensional transthoracic echocardiography [5-7]. These studies have provided only limited understanding of papillary muscle function and its relationship to mitral and ventricular function. In vivo study of papillary muscle function in humans is limited by inadequate acoustic windows in a significant number of patients and also by the inability of transthoracic echocardiography to fully delineate both papillary muscles, particularly in the long axis views, thus limiting its ability to reliably and consistently determine PM length and contractile perform- 
ance. TEE has the advantage of direct, high frequency imaging of papillary muscles without acoustic interference [8].

In patients with ischemic heart disease and dilated cardiomyopathy, we and others have provided clinical and experimental evidence linking mitral regurgitation to distortions in left ventricular geometry, papillary muscle dysfunction and alterations in the mitral and submitral apparatus and their relationships [2-5,8].In the absence of discrete valve disruption or degenerative disease, the precise mechanism of mitral regurgitation in patients with normal LV systolic function is often more difficult to ascertain. The role of papillary muscle function in this category of patients is unclear.

The specific objective of this study therefore is to provide an assessment of human papillary muscle contractile function in patients with mitral regurgitation and normal LV function using transesophageal echocardiography and to determine the relationship between papillary muscle length and function and the presence and severity of mitral regurgitation in this class of patients.

\section{Methods}

\subsection{Subjects}

The study population consisted of 85 patients meeting enrollment criteria with normal chamber dimensions and LV systolic function prospectively evaluated in our echocardiography laboratories with TEE. Fifty-one patients had isolated mitral regurgitation and thirty-four patients had no mitral regurgitation. The following exclusion criteria were applied: LVEF $<55 \%$, LV enddiastolic dimension $>56 \mathrm{~mm}$, known or suspected coronary artery disease, concomitant valve disease other than mitral regurgitation, regional wall motion abnormality, congestive heart failure, atrial fibrillation or severe systemic illness. All patients gave informed consent to TEE before the procedure. Study protocol was approved by the Institutional Review Board. Thirty-nine patients were females and forty-six were males. Mean age was $54.5 \pm 7.2$ years (range 36 - 68).

\subsection{Transesophageal Echocardiographic Imaging}

Transesophageal Echocardiographic studies were performed using HP Sonos 5500 and Vivid 3 Echo machine with a multiplane transesophageal instrument (Omniplane, Agilent and GE 6T) composed of a 64 element phased array transducer with a dual frequency feature permitting two-dimensional imaging at 5 and $3.7 \mathrm{MHz}$ The transducer array within the tip of the endoscope allowed electronic or mechanical rotation around the long axis of the ultrasound beam through a 180-degree arc. TEE was performed using standard technique. Mid papillary short axis views were obtained by advancing the probe into the mid gastric level, with retroflexion of the probe tip while the ultrasound beam was stationed at 0 degree as we have previously described $[8,9]$.

Detailed, simultaneous visualization of the long axis planes of both PM's was obtained by steering of the ultrasound beam from 0 degree to 90 degrees with lateral rotation of the probe and mild retroflexion of the probe tip. In this plane, PM images were obtained for subsequent off-line measurement of both ED and ES lengths. With gentle manipulation of the imaging probe to properly direct the ultrasound plane, precise longitudinal and short axis views of both papillary muscles were obtained at the same time throughout the cardiac cycle. Recordings were obtained in held expiration.

\subsection{Off-Line Analysis of Papillary Muscle Imaging}

Papillary muscle length was defined as the distance from the tip of the papillary muscle cone to the center of its base at the attachment of the papillary muscle to the left ventricular free wall. Papillary muscle measurements were obtained in both PM's. The longest measured length in end diastole and end systole during the same cardiac cycle was used for analysis. When more than 1 PM head was visualized, we measured the length of the 2 largest muscle groups and averaged the measurements. Mean values of 4 cardiac cycles were obtained. Percent papillary muscle fractional shortening was obtained using the following empiric formula:

$$
\frac{\text { End-Diastolic length-End-Systolic length }}{\text { End-Diastolic length }} \times 100
$$

LVEF was obtained from transthoracic echo images using the modified Simpson method. LV end-diastolic dimension was obtained from 2-D directed M mode tracing using the leading edge technique according to the American Society of Echocardiography [10].

\subsection{Quantification of Mitral Regurgitation}

The degree of severity of mitral regurgitation was quantified using 5 different methodologies (Table 1):

1. Regurgitant Fraction (RF): this is the percentage of the left ventricular stroke volume that regurgitates into the left atrium.

$$
\mathrm{RF}=\frac{V_{\text {mitral }}-V_{\text {aortic }}}{V_{\text {mitral }}} \times 100 \%
$$

where $V_{\text {mitral }}$ and $V_{\text {aortic }}$ are respectively the volumes of 
Table1. Severity of Mitral Regurgitation.

\begin{tabular}{lc}
\hline severity & severe \\
\hline RF & $>50$ \\
EROA & $>0.4 \mathrm{~cm}^{2}$ \\
Vena Contracta & $>0.7 \mathrm{~cm}$ \\
PISA Radius & $>1 \mathrm{~cm}$ \\
RJA/LAA & $>40 \%$ \\
\hline
\end{tabular}

Quantification of mitral regurgitation severity with different methods as described above.

blood that flow forward through the mitral valve and aortic valve during a cardiac cycle.

We determined forward flow through the mitral valve (from the left atrium to the left ventricle) during ventricular diastole and compared it with the flow out of the left ventricle through the aortic valve in ventricular systole. Regurgitant fraction $>50 \%$ was classified as severe mitral regurgitation.

2. Effective regurgitant orifice area (EROA): We calculated the effective regurgitant orifice area using the instantaneous regurgitant flow. The formula was derived from the following:

$$
\begin{aligned}
& V_{\text {aliasing }} * 2 \pi r^{2}=V_{\max } * \text { EROA } \\
& \text { Hence }: \text { EROA }=\operatorname{PISA}\left(2 \pi r^{2}\right) * V_{\text {aliasing }} / V_{\max }
\end{aligned}
$$

The aliasing velocity was adjusted to identify a flow convergence region with a hemispheric shape. The radius of this hemisphere was then measured, and flow rate $(\mathrm{ml} / \mathrm{s})$ was calculated as the product of the surface area of the hemisphere $\left(2 \pi r^{2}\right)$ and the aliasing velocity $(V a)$. $V_{\max }$ is the peak velocity of the regurgitant jet by continuous wave $(\mathrm{CW})$ Doppler. Effective regurgitant orifice area $>0.40 \mathrm{~cm}^{2}$ was classified as severe mitral regurgitation.

3. Vena contracta width: This was determined as the narrowest region of regurgitant jet just below the valve in LA. We measured the vena contracta width in the parasternal long axis view from transthoracic echo, in a plane perpendicular to mitral leaflet closure. Vena contracta width $>0.7 \mathrm{~cm}$ was classified as severe MR

4. PISA radius: We determined the area of the re- gurgitant flow at the level of the mitral valve by measuring the proximal isovelocity area (PISA) or zone of flow convergence. The radius of the proximal isovelocity area was determined. Regurgitation was classified as severe if PISA radius was more than $1 \mathrm{~cm}$.

5. RJA/LAA: We evaluated the regurgitant jet area (RJA) relationship to left atrial area (LAA) as previously described by Helmcke et al. (11). Severe MR was defined as RJA/LAA ratio $>40 \%$.

Study subjects meeting 4 out of 5 criteria were classified as having severe mitral regurgitation for the purpose of this investigation.

\subsection{Statistics}

All measurements were expressed as mean \pm SD. The Fisher paired $t$-test was used to evaluate differ- ences between groups. Significance level was set at a level of alpha $=0.05$.

\subsection{Intraobserver and Interobserver Agreement}

We validated our measurements with both intraobserver and interobserver variability. In order to assess intraobserver variation, we verified measurements of the end-diastolic and end-systolic lengths of both papillary muscles at two different times by the same investigator from videotaped recordings from 3 randomly selected subjects. The same measurements were again obtained on a separate occasion, in the same cardiac cycle by two independent observers (DB and ECM), using the same sample population and methods. There was excellent intraobserver consistency for either papillary muscle length $(r:+0.92, y=0.97 x+3.2, \mathrm{SEE}=2.1 \mathrm{~mm})$. Interobserver agreement was equally good $(r=0.88, y=$ $0.94 x+0.33$, SEE $1.6 \mathrm{~mm}$ ). High correlation between the two independent measurements (intra or interobserver) was noted.

\section{Results}

Out of the eighty-five patients with normal LV chamber dimensions and ejection fraction, meeting enrollment criteria, we identified mitral regurgitation in 51 patients, 28 with mild or moderate mitral regurgitation and 23 with severe mitral regurgitation. Thirty-four patients in our series had no mitral regurgitation and served as normal controls. When compared to the group without MR, there was no observed difference in ejection fraction $(61.4 \% \pm 4.3 \%$ Vs $62.7 \% \pm 6.5 \%)$ or papillary muscle percent fractional shortening $(21.7 \% \pm 3.6 \% \mathrm{Vs} 22.6 \% \pm$ $5.4 \%(\mathrm{APM})$ and $18.7 \% \pm 4.6 \%$ Vs $19.5 \% \pm 3.8 \%$ (PPM). LV chamber dimensions (ED and ES) were statistically similar between the groups. The only observed difference between the groups was in the significantly longer anterior papillary muscle length in those with mitral regurgitation compared to those without mitral regurgitation at $\mathrm{ED}(p=0.008)$ and ES $(p=0.04)$. These differences were more pronounced in those with severe mitral regurgitation (Tables 2A \& B).

\section{Discussion}

Human investigations of papillary muscle function and relevance have been limited by small number of subjects, as well as the inherent difficulty of obtaining sufficiently clear images of papillary muscles with transthoracic im- 
Table 2A. Comparison between groups without MR and those with MR.

\begin{tabular}{lccc}
\hline & no MR $(\boldsymbol{n}=\mathbf{3 4})$ & MR $(\boldsymbol{n}=\mathbf{5 1})$ & $\boldsymbol{p}$-value \\
\hline LVEF & $62.7 \pm 6.5$ & $61.4 \pm 4.3$ & $\mathrm{NS}$ \\
Anterior PM & & & \\
ED length & $2.88 \pm 0.47$ & $3.38 \pm 0.61$ & 0.008 \\
ES length & $2.17 \pm 0.33$ & $2.46 \pm 0.51$ & 0.04 \\
PMFS \% & $22.6 \pm 5.4$ & $21.7 \pm 3.6$ & $\mathrm{NS}$ \\
Posterior PM & & & \\
ED length & $2.49 \pm 0.47$ & $2.58 \pm 0.69$ & $\mathrm{NS}$ \\
ES length & $1.92 \pm 0.37$ & $1.89 \pm 0.67$ & $\mathrm{NS}$ \\
PMFS\% & $19.5 \pm 3.8$ & $18.7 \pm 4.6$ & $\mathrm{NS}$ \\
\hline
\end{tabular}

Statistically significant increase in the length (ED \& ES) of the anterior papillary muscle is noted in patients with MR compared to those without MR and no observed difference was noted between the two groups in PMFS \& LVEF. ED, end diastole; ES, end systole; MR, mitral regurgitation; LVEF, left ventricular ejection fraction; PMFS, papillary muscle fractional shortening; PM, papillary muscle.

Table 2B. Comparison between groups without MR and those with Severe MR.

\begin{tabular}{lccc}
\hline & no MR $(\boldsymbol{n}=\mathbf{3 4})$ & $\begin{array}{c}\text { Severe MR } \\
(\boldsymbol{n}=\mathbf{2 3})\end{array}$ & $\boldsymbol{p}$-value \\
\hline LVEF & $62.7 \pm 6.5$ & $60.5 \pm 9.3$ & NS \\
Anterior PM & & & \\
ED length & $2.88 \pm 0.47$ & $3.73 \pm 0.85$ & 0.002 \\
ES length & $2.17 \pm 0.33$ & $2.87 \pm 0.71$ & 0.004 \\
PMFS \% & $22.6 \pm 5.4$ & $20.3 \pm 6.8$ & NS \\
Posterior PM & & & \\
ED length & $2.49 \pm 0.47$ & $2.87 \pm 0.91$ & NS \\
ES length & $1.92 \pm 0.37$ & $2.08 \pm 0.65$ & NS \\
PMFS\% & $19.5 \pm 3.8$ & $18.4 \pm 6.9$ & NS \\
\hline
\end{tabular}

Increase in the length (ED \& ES) of the anterior papillary muscle is more pronounced in patients with severe MR and no observed difference was noted between the two groups in PMFS \& LVEF. ED, end diastole; ES, end systole; MR, mitral regurgitation; LVEF, left ventricular ejection fraction; PMFS, papillary muscle fractional shortening; PM, papillary muscle.

aging $[5,7,12,13]$. Using the superior imaging capabilities of transgastric echocardiography, we evaluated the relationship between papillary muscle function and mitral regurgitation in patients with normal LV chamber size and function and mitral regurgitation. Over the past few decades, progressive degenerative disease of the mitral valve and its apparatus has assumed increased prominence as a cause of mitral regurgitation. Also increasingly recognized since the early eighties is mitral regurgitation from disruption of valve leaflets mainly from infectious endocarditis or other systemic illnesses. Significant MR without intrinsic leaflet pathology may be encountered in $11 \%$ of patients [14]. In those patients without evidence of disruptive or degenerative process of the valve and its supporting structures, the precise mechanism of mitral regurgitation remains less certain. Several etiologic mechanisms for functional mitral regurgitation have been proposed in those patients with dilated cardiomyopathy and ischemic heart disease. There is a strong evidence to suggest that ischemic heart disease or dilated cardiomyopathy may incite significant mitral regurgitation by causing displacement or tethering of mitral leaflets (resulting in impaired leaflet coaptation), through several mechanisms such as increased annular dilatation, volume overload, and reduced myocardial contractility and alterations in left ventricular geometry [4,15-22]. These proposed mechanisms cannot be applied to those patients with normal LV chamber dimensions and function and with no objective evidence of ischemia or coronary artery disease. The precise mechanism of mitral regurgitation in this population remains unresolved and controversial.

Papillary muscle dysfunction has previously been proposed as a mechanistic cause of mitral regurgitation. We have previously shown that papillary muscles make significant contribution to ventricular ejection mechanics in humans [9].However, the role of papillary muscle dysfunction in the causation of mitral regurgitation in the presence of normal LV systolic function is less clear and the subject of ongoing debate. There is indeed experimental evidence to suggest that isolated papillary muscle dysfunction in an adequately contractile ventricle is not associated with significant mitral regurgitation [3]. In an animal model of experimental mitral regurgitation, Kaul et al. showed that ischemic mitral regurgitation was not related to papillary muscle dysfunction, but rather to global LV myocardial ischemia [3].

Messas et al. [23] in animals, using 3D echocardiography and Uemura et al. [24] in humans using transthoracic echocardiography demonstrated that the severity of ischemic MR is primarily related to papillary muscle tethering distance and does not have any consistent relationship with papillary muscle dysfunction itself. In fact it has been shown that PM dysfunction per se, is associated with systolic elongation or less systolic shortening and paradoxically may result in attenuation of leaflet tethering and ischemic MR.

In a human study using transthoracic echocardiography, Kisanuki et al. [7] found no correlation between mild mitral regurgitation and papillary muscle dysfunction. Our work on humans with TEE extends these earlier observations and demonstrates for the first time, the absence of correlation between papillary muscle function and mitral regurgitation in those patients with normal LV chamber size and function. Rather, we show that the 
length of the anterior mitral valve leaflet is a major predictor of the presence of mitral regurgitation in this class of patients. We believe that the spatial relationship between the papillary muscles, the chordae and the mitral valve leaflets are crucial determinants of the occurrence of mitral regurgitation. Any alteration in the spatial relationship between the papillary muscles and the mitral apparatus may result in significant mitral regurgitation. Such aberrations could result from an abnormal elongation of the papillary muscle, LV dilatation or ischemia resulting in abnormal coaptation of the valve leaflets and hence mitral regurgitation. Our findings indicate that in patients with normal LV function and mitral regurgitation, anterior papillary muscle elongation plays a significant role in the causation of functional mitral regurgitation. We speculate that the abnormal elongation in anterior PM length in these patients may result in disruption of the spatial relationship between the PM and the MV leaflets resulting in impaired leaflet coaptation and mitral regurgitation. In the 51 patients in our series with normal chamber dimensions, LV function and mitral regurgitation, PM fractional shortening was normal and similar between the groups and could not separate those patients with mild mitral regurgitation from those with more severe mitral regurgitation.

\section{REFERENCES}

[1] A. K. Mittal, M. Langston Jr, A. Seltzer and W. J. Kerth, "Combined Papillary Muscle and Left Ventricular Dysfunction as a Cause of Mitral Regurgitation: An Experimental Study," Circulation, Vol. 44, No. 2, 1971, pp. 174-180.

[2] A. G. Tsakiris, G. C. Rastelli, D. D. Amorim, J. L. Titus and E. Wood, "Effect of Experimental Papillary Muscle Damage on Mitral Valve Closure in Intact Anesthetized Dogs," Mayo Clinic Proceedings, Vol. 45, No. 4, 1970, pp. 275-285.

[3] S. Kaul, W. D. Sponitz, W. P. Glasheen and D. A. Touchstone, "Mechanism of Ischemic Mitral Regurgitation: An Experimental Evaluation," Circulation, Vol. 84, No. 5, 1991, pp. 2167-2180.

[4] T. Kono, H. N. Sabbah, H. Rosman, A. Moshin, J. Syed, P. D. Stein and S. Goldstein, "Mechanism of Functional Mitral Regurgitation During Myocardial Ischemia," Journal of the American College of Cardiology, Vol. 19, No. 5, 1992, pp. 1101-1105. doi:10.1016/0735-1097(92)90302-4

[5] E. C. Madu and I. A. D'Cruz, "The Vital Role of Papillary Muscles in Mitral and Ventricular Function: Echocardiographic Insights," Clinical Cardiology, Vol. 20, No. 2, 1997, pp. 93-98. doi:10.1002/clc.4960200203

[6] P. C. Come, M. F. Riley, R. Weintraub, J. P. Morgan and S. Nakao, "Echocardiographic Detection of Complete and
Partial Papillary Muscle Rupture during Acute Myocardial Infarction," The American Journal of Cardiology, Vol. 56, No. 12, 1985, pp. 787-789. doi:10.1016/0002-9149(85)91137-3

[7] A. Kisanuki, Y. Otsuji, R. Kuroiwa, T. Murayama, R. Matsushita, K. Shibata, T. Yutsudo, S. Nakao, K. Nomoto et al., "Two Dimensional Echocardiographic Assessment of Papillary Muscle Contractility in Patients with Prior Myocardial Infarction," Journal of the American College of Cardiology, Vo. 21, No. 4, 1993, pp. 932-938. doi:10.1016/0735-1097(93)90350-A

[8] E. C. Madu, D. S. Baugh, I. A. Cruz, C. Johns, "Left Ventricular Papillary Muscle Morphology and Function in Left Ventricular Hypertrophy and Left Ventricular Dysfunction," Medical Science Monitor, Vol. 7, No. 6, 2001, pp. 1212-1218.

[9] E. C. Madu, D. S. Baugh, C. Johns and I. A. D'Cruz, "Papillary Muscle Contribution to Ventricular Ejection in Normal and Hypertrophic Ventricles: A Transesophageal Echocardiographic Study," Echocardiography, Vol. 18, No. 8, 2001, pp. 633-638. doi:10.1046/j.1540-8175.2001.00633.x

[10] D. J. Sahn, A. DeMaria, J. Kisslo and A. Weyman, "Recommendations Regarding Quantitation in M-mode Echocardiography: Results of a Survey of Echocardiographic Measurements," Circulation, Vol. 58, No. 6, December 1978, pp. 1072-1083.

[11] F. Helmcke, N. C. Nanda, M. C. Hsiung, B. Soto, C. K. Adey, R. G. Goyal and R. P. Gate Wood Jr, "Color Doppler Assessment of Mitral Regurgitation with Orthogonal Planes," Circulation, Vol. 75, No. 1, 1975, pp. 175-183.

[12] C. M. Boltwood, C. Tei, M. Wong and P. M. Shah, "Quantitative Echocardiography of the Mitral Complex in Dilated Cardiomyopathy: The Mechanism of Functional mitral Regurgitation," Circulation, Vol. 68, No. 3, 1983, pp. 498-508.

[13] A. F. Grimm, B. L. Lendrum and L. Hum-Lin, "Papillary Muscle Shortening in the Intact Dog: Cine Radiographic Study of Tranquilized Dogs in the Upright Position," Circulation Research, Vol. 36, No. 1, 1975, pp. 49-57.

[14] W. Mazur and S. Nagueh, "Echocardiographic Evaluation of Mitral Regurgitation," Current opinion in Cardiology, Vol. 16, No. 4, 2001, pp. 246-250. doi:10.1097/00001573-200107000-00005

[15] Y. Otsuji, M. D. Handschumacher, E. Schwammenthal, L. Jiang, J. K. Song, J. L. Guerrero, G. J. Vlahakes and R. A. Levine, "Insights from Three-Dimensional Echocardiography into the Mechanism of Functional Mitral Regurgitation: Direct in vivo Demonstration of Altered Leaflet Tethering Geometry," Circulation, Vol. 96, No. 6, 1997, pp. 1999-2008.

[16] S. He, A. A. Fontaine, E. Schwammenthal, A. P. Yoganathan and R. A. Levine. "Integrated Mechanism for Functional Mitral Regurgitation: Leaflet Restriction Versus Coapting Force: in vitro Studies," Circulation, Vol. 96, No. 6, 1997, pp. 1826-1834.

[17] S. F. Yiu, M. Enriquez-Sarano, C. Tribouilloy, J. B. Sew- 
ard and A. J. Tajik, "Determinants of the Degree of Functional Mitral Regurgitation in Patients with Systolic Left Ventricular Dysfunction: A Quantitative Clinical Study," Circulation, Vol. 102, No. 2, 2000, pp. 1400-1406.

[18] Y. Otsuji, Handschumacher, N. Liel-Cohen, H. Tanabe, L. Jiang, E. Schwammenthal, J. L. Guerrero, L. A. Nicholls, G. J. Vlahakes, R. A. Levine et al. "Mechanism of Ischemic Mitral Regurgitation with Segmental Left Ventricular Dysfunction: Three-Dimensional Echocardiographic Studies in Models of Acute and Chronic Progressive Regurgitation," Journal of the American College of Cardiology, Vol. 37, No. 2, 2001, pp. 641-648. doi:10.1016/S0735-1097(00)01134-7

[19] R. C. Gorman, J. S. McCaughey, M. B. Radcliffe, K. B. Gupta, J. T. Streicher, V. A. Ferrari, St. M. G. John-Sutton, D. K. Bogen, L. H. Edmunds Jr., "Pathogenesis of Acute Ischemic Mitral Regurgitation in Three Dimensions," The Journal of Thoracic and Cardiovascular Surgery, Vol. 109, No. 4, 1995, pp. 684-693. doi:10.1016/S0022-5223(95)70349-7

[20] F. A. Tibayan, F. Rodriguez, M. K. Zasio, L. Bailey, D. Liang, G. T. Daughters, F. Langer, N. B. Ingels Jr and D. C. Miller. "Geometric Distortions of the Mitral Valvular Ventricular Complex in Chronic Ischemic Mitral Regurgitation," Circulation, Vol. 108, No. 10, 2003, pp. II-116II-121.

[21] M. Komeda, J. R. Glasson, A. F. Bolger, G. T. Daughters,
A. Mac Isaac, S. N. Oesterle, N. B. Ingels Jr. and D. C. Miller, "Geometric Determinants of Ischemic Mitral Regurgitation," Circulation, Vol. 96 (9 Suppl), 1997, pp. II-128-33.

[22] J. Kwan, T. Shiota, D. A. Agler, Z. B. Popovic, J. X. Qin, M. A. Gillinov, W. J. Stewart, D. M. Cosgrove, P. M. McCarthy and J. D. Thomas, "Geometric Differences of the Mitral Apparatus between Ischemic and Dilated Cardiomyopathy with Significant Mitral Regurgitation: Real-Time Three-Dimensional Echocardiography Study," Circulation, Vol. 107, No. 8, 2003, pp. 1135-1140. doi:10.1161/01.CIR.0000053558.55471.2D

[23] E. Messas, J. L. Guerrero, M. D. Handschumacher, C. M. Chow, S. Sullivan, E. Schwammenthal and R. A. Levine, "Paradoxic Decrease in Ischemic Mitral Regurgitation with Papillary Muscle Dysfunction; Insight from ThreeDimensional and Contrast Echocardiography with Strain Rate Measurement," Circulation, Vol. 104, No. 16, 2001, pp. 1952-1957. doi:10.1161/hc4101.097112

[24] T. Uemura, Y. Otsuji, K. Nakashiki, S. Yoshifuku, B. Yu, N. Mizukami, E. Kuwahara, S. Hamasaki, A. Kisanuki, S. Minagoe, R. A. Levine and C. Tei, "Papillary Muscle Dysfunction Attenuates Ischemic Mitral Regurgitation in Patients with Localized Basal Inferior Left Ventricular Remodeling," The Journal of Thoracic and Cardiovascular Surgery, Vol. 46, No. 1, 1995, pp. 113-119. 